Journal of Thermal Science Vol.16, No.3 $1-7$

Paper reference: ISAIF8-0053

\title{
Modeling of 3-D Losses and Deviations in a Throughflow Analysis Tool
}

\author{
Jean-François Simon ${ }^{1}$ Olivier Léonard ${ }^{2}$ \\ 1. Techspace Aero - Aerodynamic Group jsimon@techspace-aero.be \\ 2. University of Liège - Turbomachinery Group o.leonard@ulg.ac.be
}

\begin{abstract}
This contribution is dedicated to the modeling of the end-wall flows in a throughflow model for turbomachinery applications. The throughflow model is based on the Euler or Navier-Stokes equations solved by a Finite-Volume technique. Two approaches are presented for the end-wall modeling. The first one is based on an inviscid formulation with dedicated 3-D distributions of loss coefficient and deviation in the end-wall regions. The second approach is directly based on a viscous formulation with no-slip boundary condition along the annular end-walls and blade force modification in the region close to the end-walls. The throughflow results are compared to a series of 3-D Navier-Stokes calculations averaged in the circumferential direction. These 3-D calculations were performed on the three rotors of a low pressure axial compressor, for a series of tip clearance values.
\end{abstract}

Keywords: throughflow, losses, deviations, axial compressors

\section{Introduction}

The throughflow level of approximation still remains an important tool for designing turbomachines [2] even though 3-D calculations are used more and more early in the design process. The throughflow models heavily rely on empirical inputs such as 2-D correlations or end-wall loss models. This feature has shown to be efficient but lack of generality.

Two-dimensional empirical correlations are available in the literature but they have generally been designed for classical families of blade profiles such as $\mathrm{C} 4$ or NACA-65 and are not directly applicable to the custom-based profiles nowadays used in the industry. However, it is possible to adapt existing loss correlations to modern blade profiles [5] or to derive correlations for an arbitrary profiles with the help of blade-to-blade codes [6].

Concerning the 3-D losses, very few results are available in the open literature. The main contribution on the compressor side is from Roberts et al. [7,8]. They proposed correlations based on collected empirical data to build up 3-D distributions of loss coefficient and deviation in the end-wall regions. A throughflow analysis tool based on an inviscid model, supplemented with these 3-D correlations for the end-wall losses is the first approach studied here.

The second approach presented in this contribution is directly based on the Navier-Stokes equations with blade force modifications close to the end-walls to capture the end-wall flows. By including more physics in the model, less empiricism is needed and a more general method can therefore be devised.

The objective of the present work is to test both approaches for the modeling of the end-wall flows within a throughflow model. The results are compared to a series of 3-D Navier-Stokes calculations performed on axial compressor rotors.

\section{Throughflow analysis tool}

The throughflow analysis tool used in the present work is based on the Euler equations $[1,11]$ and the $\mathrm{Na}$

Received: December 2006

Jean-François Simon: Professor 


\begin{tabular}{|c|c|c|c|}
\hline Nomenclature & & $\varpi$ & loss coefficient \\
\hline $\mathrm{AR}$ & channel aspect ratio, $\mathrm{h} / \mathrm{s}$ & $\theta$ & flow deflection \\
\hline $\mathrm{c}$ & mean blade chord & $R=W_{2} / W_{1}$ & velocity ratio \\
\hline $\mathrm{h}$ & mean span or mean blade height & Subscripts & \\
\hline $\mathrm{s}$ & blade spacing & 1 & inlet \\
\hline $\bar{S}_{3 D}$ & extent of 3-D loss, in fraction of span & 5 & $5 \%$ of span from tip at the blade outlet \\
\hline$\left(\bar{S}_{3 D}\right)_{\max }$ & location of max. 3-D loss, in fraction of span & 95 & $\begin{array}{l}95 \% \text { of span from tip at the blade out- } \\
\text { let }\end{array}$ \\
\hline$\overline{T C}$ & rotor blade tip clearance, in fraction of span & $2 \mathrm{D}$ & relative to a two-dimensional flow \\
\hline Greek letters & & $3 \mathrm{D}$ & relative to a three-dimensional flow \\
\hline$\delta$ & flow deviation angle & $\mathrm{h}$ & hub \\
\hline$\delta^{*}$ & displacement thickness & $\max$ & maximum location \\
\hline$\sigma$ & solidity, $c / s$ & mo & maximum overturning location \\
\hline$\phi$ & blade camber angle & $\mathrm{rt}$ & rotor tip \\
\hline
\end{tabular}

vier-Stokes equations [9]. Such models allow to calculate the flow field whatever the flow regime is, they are able to perform transient simulations and they can cope with 2-D flow recirculations. These approaches offer alternatives to the classical streamline curvature method.

Details on the present solver are available in [9]. In short, the equations are solved by a finite volume technique. The inviscid fluxes are computed thanks to the Roe scheme and the viscous flux by the diamond path scheme. The time integration is performed by an explicit multistep Runge-Kutta scheme.

The turbulence model is of the mixing length type and a radial mixing model based on a turbulent diffusion assumption is used. The inviscid blade force is computed by an additional time-dependent equation [1], while the viscous blade force is calculated by a distributed loss model and empirical correlations. In order to avoid loss generation by the inviscid blade force, its direction is chosen orthogonal to the flow field. An adaptation of the inviscid blade force in the end-wall regions is therefore required. More details on these modifications are available in the following sections.

\section{3-D database}

The test-case used in the present work is composed of 3 rotors of a low pressure compressor designed by Techspace Aero. For each rotor, four different tip clearance values were considered: $0.4,0.8,1$ and 1.2 percents of the mean blade height. The database is composed of 3-D Navier-Stokes calculation results. For each configuration, two computations were run, one with flow conditions and blade incidence representative of an on-design configuration, a second one representative of near-stall conditions. The mesh is made of about 1.2 million grid points and the turbulence model is algebraic.

These 3-D Navier-Stokes computations, after a circumferential averaging, provide the boundary conditions for the throughflow calculations. Rotor blades were discretized in ten sections at different blade heights to transfer the blade camber and thickness to the throughflow analysis tool in a rather complete way. The radial blade forces were neglected.

\section{Inviscid annulus end-walls formulation}

The first approach for modeling the end-wall flows is based on an inviscid treatment of the hub and shroud with a slip condition. The effect of the viscous flow developing along the end-walls is (partly) reintroduced by adding a 3-D distribution of deviation and loss coefficient on the top of the 2-D distributions:

$$
\begin{aligned}
\varpi & =\varpi_{2 D}+\varpi_{3 D} \\
\delta & =\delta_{2 D}+\delta_{3 D}
\end{aligned}
$$

Figure 1 illustrates the 3 -D distributions obtained with Roberts correlations [7,8] for a rotor. The usual evolution of the deviation with an overturning at the hub and an overturning followed by an underturning at the tip is observed. The loss coefficient distribution also shows a classical shape at both end-walls.

The expression of Roberts correlations is reproduced hereafter in order to highlight the main parameters driving the loss and deviation generation.

Correlations for 3D losses at shroud :

$$
\begin{gathered}
\left(\varpi_{3 D}\right)_{\max , r t}=0.25 \tanh \sqrt{10^{3} \bar{\delta}_{1}^{*} \cdot \overline{T C}} \\
\left(\bar{S}_{3 D}\right)_{\max , r t}=\frac{\left(\varpi_{3 D}\right)_{\max , r t}}{2} \\
\left(\bar{S}_{3 D}\right)_{r t}=2.5\left(\bar{S}_{3 D}\right)_{\max , r t}
\end{gathered}
$$

Correlations for 3D losses at hub :

$$
\left(\varpi_{3 D}\right)_{\max }=0.2 \tanh \sqrt{15\left(\frac{\phi \cdot \bar{\delta}_{1}^{* 2}}{A R \cdot \sqrt{\sigma}}\right)}
$$




$$
\begin{gathered}
\left(\bar{S}_{3 D}\right)_{\max }=0.1 \\
\bar{S}_{3 D}=2.5 \cdot\left(\bar{S}_{3 D}\right)_{\max }=0.25
\end{gathered}
$$

Correlations for $3 \mathrm{D}$ deviations at hub :

$$
\begin{gathered}
\bar{\Delta}_{95}=-2 \\
\bar{h}_{h}=0.85
\end{gathered}
$$

Correlations for 3D deviations at shroud :

$$
\begin{gathered}
\bar{\Delta}_{5}=30 \tanh \left(10^{3} \bar{\delta}_{1}^{*} \cdot \overline{T C}\right) \\
\bar{\Delta}_{m o}=-1.25 \\
\bar{h}_{m o}=0.15
\end{gathered}
$$

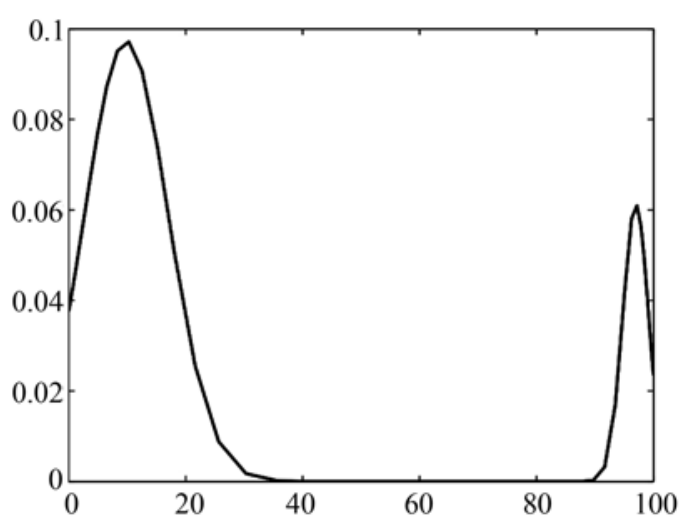

(a) Loss coefficient vs blade height

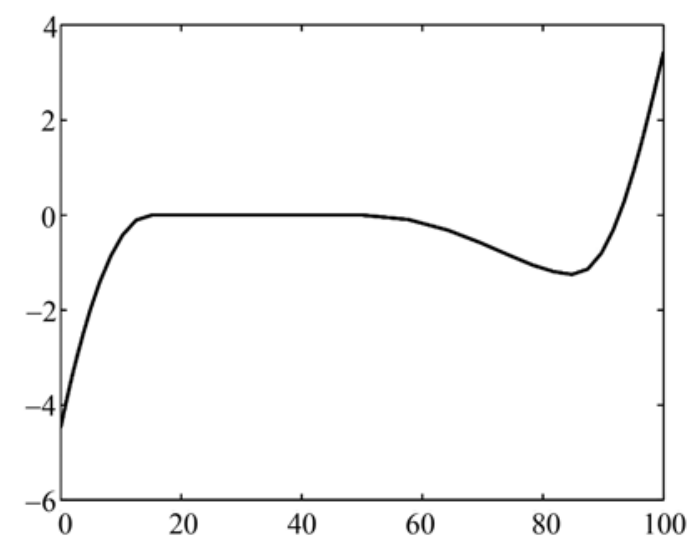

(b) Deviation vs blade height

Figure 1: Original 3-D correlations of Roberts

These correlations allow to describe the distributions of the 3-D loss coefficients and deviations using only a few parameters, the latter being obtained from the flow-path geometry, the blade geometry and the inlet boundary layer displacement thickness. These parameters however do not allow to obtain the whole distributions along the span but only some typical points of these distributions. Additional information must be provided. The following curves (Gaussian law, 1-D damped oscillator equation and parabola) have been used to connect the points provided by the correlation of Roberts:

$$
\begin{gathered}
f(x)=\frac{1}{\sigma \sqrt{2 \pi}} \exp \left(-\frac{(x-\mu)^{2}}{2 \sigma^{2}}\right) \\
f(x)=\exp \left(\frac{x}{\tau}\right)\left[f_{0} \cos \left(\omega_{d} x\right)+\beta \sin \left(\omega_{d} x\right)\right] \\
f(x)=a x^{2}+b x+c
\end{gathered}
$$

A last remark has to be added before presenting the results obtained with this approach. The loss coefficient in the tip region obtained from the 3-D Navier-Stokes computations does not always follow the classical distribution shown on Figure 1(a). Distributions with no peak are obtained when the value of the tip clearance is low compared to the boundary layer thickness, as shown on Figure 2, which gives the loss coefficient distribution for different tip clearance values, for one of the rotors considered in this work. These distributions with no peak have not been included in the database. Clearly, another way to characterize the loss coefficient distribution for low tip clearance values is needed.

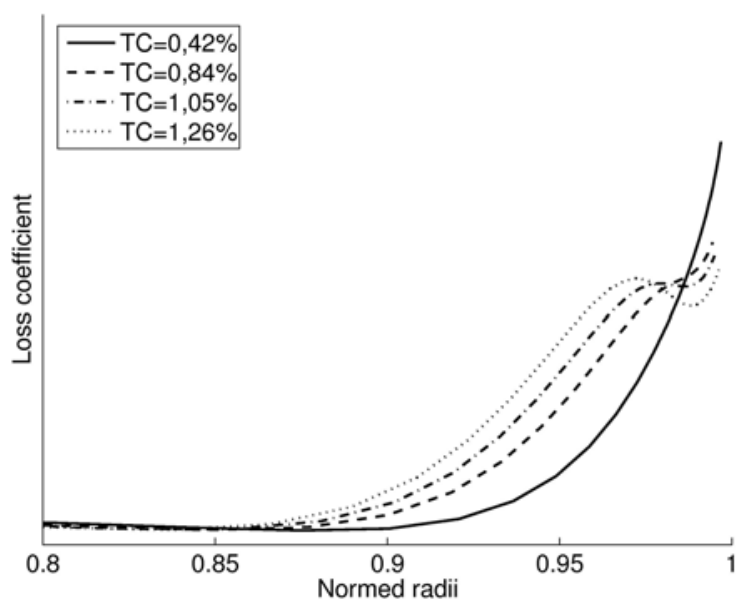

Figure 2: Radial distribution of loss coefficient obtained from $3 \mathrm{D}$ calculations

Applying the original correlations provided by Roberts to the rotors considered in the present work failed to reproduce the averaged 3-D Navier-Stokes results. A calibration procedure was performed on the parameters of Roberts correlations so as to fit the 3-D Navier-Stokes database corresponding to the first rotor blade. Figure 3 compares the loss coefficient distributions in the tip region obtained with the original correlation, with the calibrated one and with the 3-D averaged one.

Unfortunately applying this calibrated correlation to the other two rotor geometries failed to reproduce the flow characteristics for these rotors, as shown on Figure 4. This figure shows one of the parameters of Roberts correlation, $\left(\varpi_{3 D}\right)_{\max , r t}$, i.e. the peak in the 3-D loss coefficient, versus a combination of the clearance gap 
and the boundary layer thickness. These results are related to on-design operating points only.

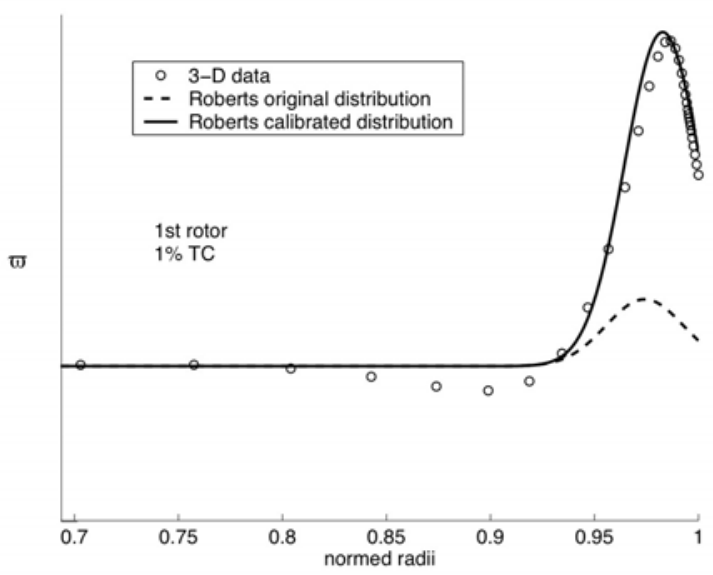

Figure 3: Loss coefficient distribution at rotor tip

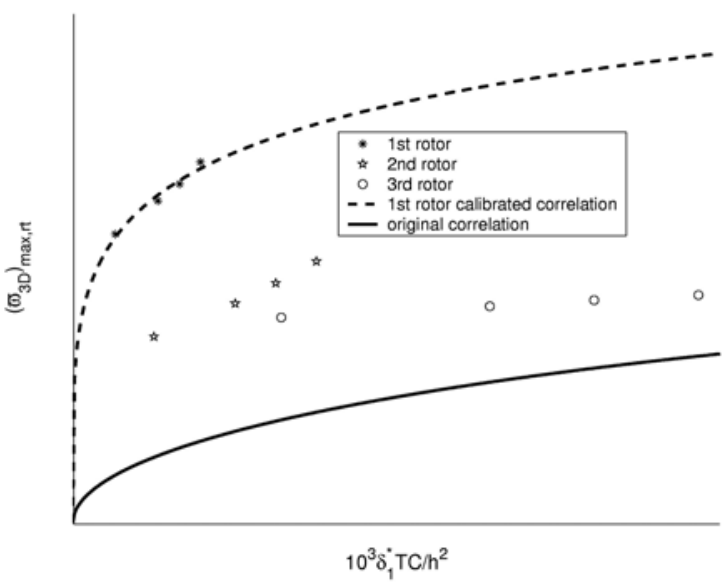

Figure 4: Clearance effect fitted on 1st rotor data

The above results demonstrate the need for including extra parameters in the Roberts correlation in order to take into account additional effects such as the blade loading. Indeed the main drawback of Roberts correlations is that they do not rely on quantities representative of the aerodynamic loading (to the exception of the blade camber for the maximum loss at hub).

From that observation, it was decided to test several functions depending on the loading, the velocity ratio or the flow deflection in order to derive the sought correlations. This was performed systematically for each variable to be modeled by the correlations. The obtained results are presented hereafter.

Figure 5 illustrates the results obtained for the peak of the loss coefficient distribution in the tip region, for several rotors and several clearance values. The function used to approximate it is of the following form :

$$
\left(\varpi_{3 D}\right)_{\max , r t}=A \tanh \left(R^{a} \cdot \theta^{b}\left(1000 \cdot \bar{\delta}_{1}^{*} \cdot \overline{T C}\right)\right)
$$

where $A, a$ and $b$ are the parameters determined by the calibration process. The results are thought to be good. The 3-D Navier-Stokes distributions for which no peaks are observed have also been added on the same figure. For these distributions the maximum of the loss coefficient has been used instead of the (non-existing) peak. These values have however not been used to build the correlations.

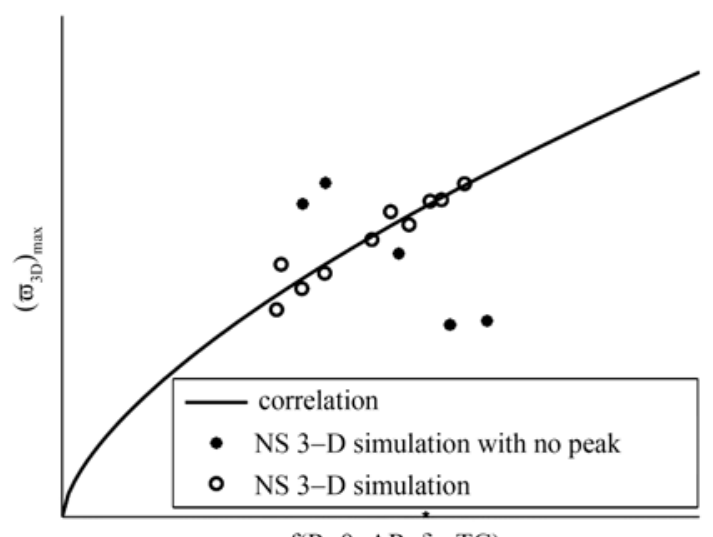

$\mathrm{f}\left(\mathrm{R}, \theta, \mathrm{AR}, \delta_{1}, \mathrm{TC}\right)$

Figure 5: Loss coefficient peak at tip

Figure 6 illustrates the same quantity for the hub region and again illustrates the quality of the results.

It is recognized that the database used here is far to be sufficiently dense to allow drawing general correlations such as those devised by Roberts.

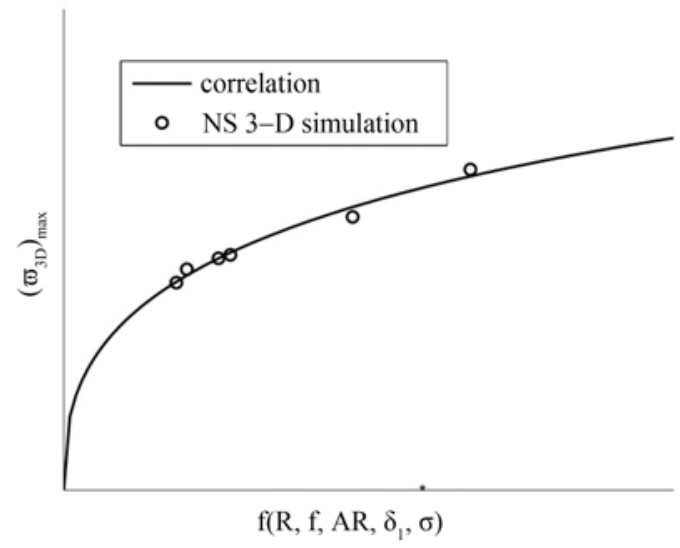

Figure 6: Loss coefficient peak at hub

More operating points and more geometries should be considered and tested. However, the proposed procedure, based on loading criterions and on 3-D numerical simulations seems to be promising and should be easily extended to a larger database.

\section{Viscous annulus end-walls formulation}

However, it must be recognized that the previous method essentially based on empirical correlations lacks 
of generality. An improved method for capturing the 3-D flows, inspired from Gallimore [4], is proposed hereafter. In the frame of its viscous Streamline Curvature method, Gallimore proposed to modify the blade force (its circumferential component more exactly) in the end-wall regions. The modification he proposed for the blade force is based on experimental observations of the blade wall static pressure field, noteworthy the observations of Dring [3]. These results show that the circumferential component of the blade force varies smoothly across the span and does not exhibit rapid changes as one approaches the end-walls contrary to the circumferential momentum jump across the blade row. This is an illustration of the well known fact that the pressure field does not vary much across a boundary layer in the direction orthogonal to the wall.

From that observation it seems possible to extrapolate some information across the span to model the blade force in the end-wall regions. A simple model for the evolution of the circumferential blade force in these regions is proposed hereafter for a rotor:

- for the hub flow, the circumferential component of the blade force is kept constant over a given portion of the span, starting from the hub,

- for the tip flow, the blade force is set to zero in the gap. The blade force is also kept constant from a given spanwise position to the tip gap. It was found that this last modification is necessary in order to obtain the correct level of underturning in the tip region. It is in agreement with the experimental observations of Gallimore [4] and Dring [3].

This is a rather simple model and more elaborated prediction of the evolution of the blade force inside the boundary layer can be devised (see the model of Gallimore [4] for example). The purpose here is to evaluate the benefit that such a method could bring on the 3-D loss and deviation predictions.

In the regions where the inviscid blade force is modified, the flow does not follow any more the camber surface. In order to avoid the generation of numerical losses, it is important that the blade force remains orthogonal to the flow. For this reason, it is not possible to work directly on the modulus of the blade force. To avoid imposing a constrain on the modulus of the force, the modification is performed on its circumferential component only. The other components are computed so that the resulting blade force is still orthogonal to the flow field.

The modification of the blade force is tested hereafter on the first rotor studied in the previous section. The flow path is shown in Figure 7, together with the mesh used in the present study.

The modification of the blade force has been performed over about $15 \%$ of the span. This extent has been chosen more or less arbitrarily in order to influence the flow field over the correct fraction of the span. This extent could be determined automatically with a criterion based on the boundary layer thickness for example.

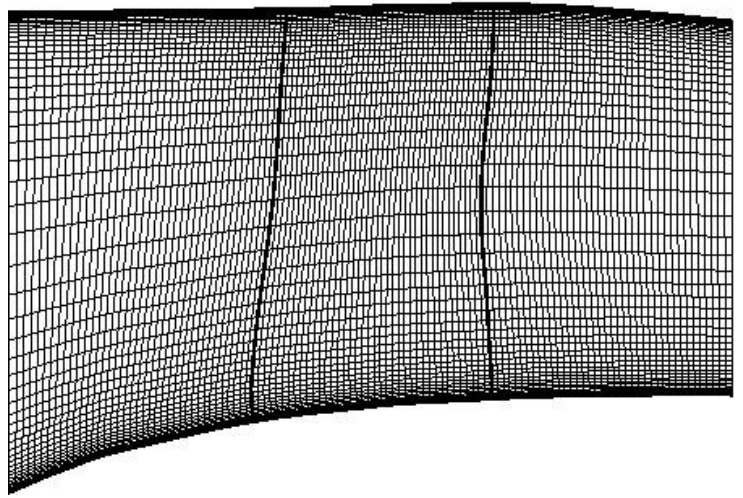

Figure 7: Typical mesh for Navier-Stokes calculation

Figures 8 and 9 present the results obtained in terms of deviation and loss coefficient distributions. For both quantities, two calculations are presented: one with the blade force modification (TF NS 3-D) and the other one without (TF NS 2-D). These two calculations are compared with the distribution obtained from the 3-D Navier-Stokes calculation which represents the reference solution.

Figure 8(a) shows the deviation in the hub region. Thanks to the constant blade force through the boundary layer, the overturning is predicted both in location and magnitude. At the tip, the underturning is also predicted but with less precision, as shown on Figure 8(b). It is globally underestimated except very close to the tip gap. A way to improve the result would be to decrease the blade force in that region instead of keeping it constant. This would be in agreement with the experimental results obtained by Storer and Cumpsty [10]. The improvement brought by the modification of the blade force compared to the 2-D solution is clearly demonstrated.

Concerning the loss coefficient, the blade force modification has only a slight effect on the flow development at the hub (Figure 9(a)). Indeed the proposed modification of the inviscid blade force does not model the loss generated by the interaction of the blade and the annulus end-wall. For the tip region (Figure 9(b)), the situation is different, as canceling the blade force in the tip gap induces some friction forces at the tip of the blade. The resulting loss coefficient is more similar to the one obtained by the 3-D Navier-Stokes simulation but with a lower intensity: some flow features such as the loss generated by the tip vortex flow is not modeled in the throughflow.

In conclusion, the 3-D deviation is relatively well predicted by the blade force modification model. This is not the case for the 3-D losses as the mechanisms that generate those losses are not modeled (at the exception of 
some parts for the tip region). Away to model these losses would be to rely on the characteristics of the captured 3-D deviation. Indeed, as it is (partly) the same phenomena that are responsible for the generation of the 3-D losses and the 3-D deviation, it should be possible to determine the 3-D loss coefficient from the captured 3-D deviation.

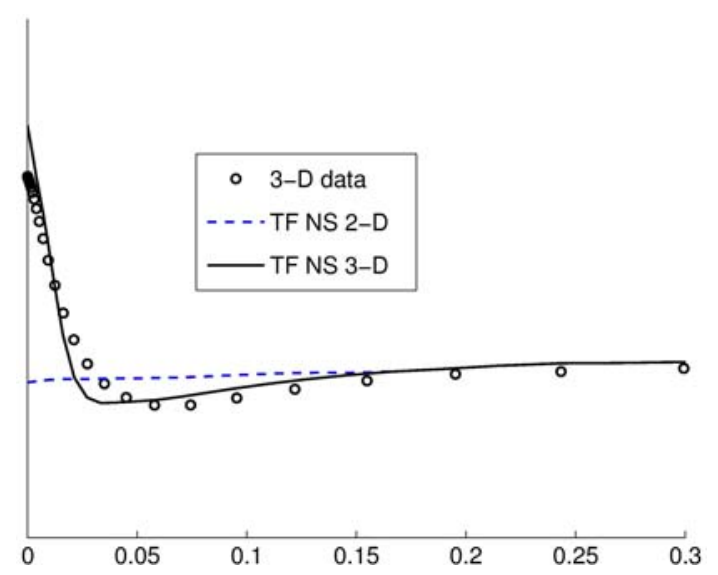

(a) deviation at hub vs normed radii

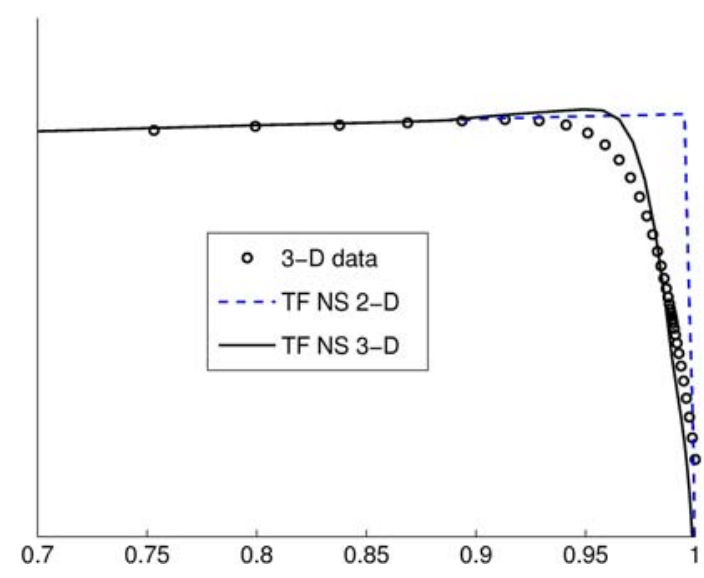

(b) deviation at tip vs normed radii

Figure 8: Impact on the deviation of the blade force modifications in the end-wall regions

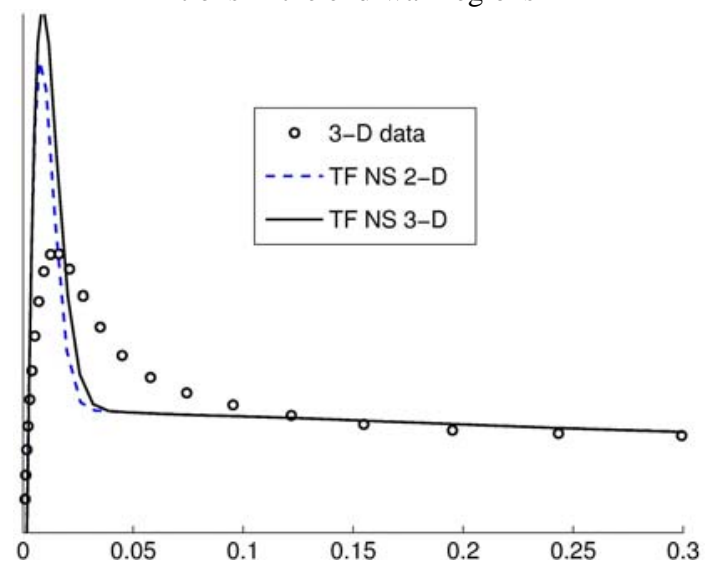

(a) loss coefficient at hub vs normed radii

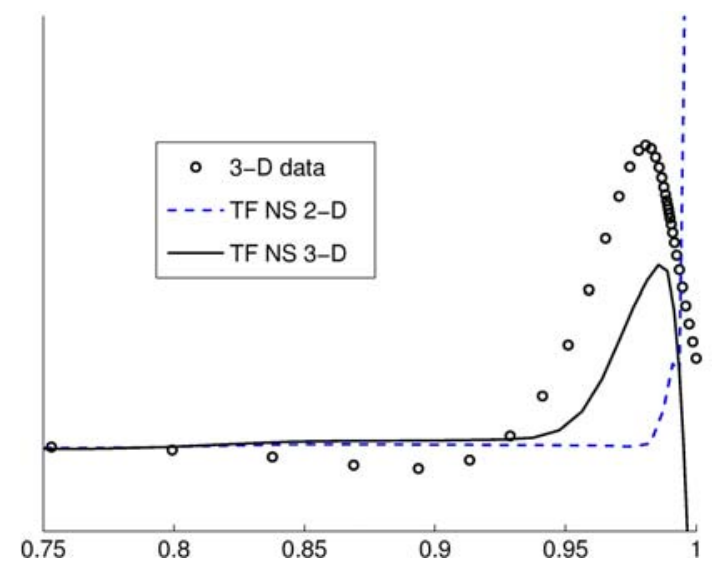

(b) loss coefficient at tip vs normed radii

Figure 9: Impact on the loss coefficient of the blade force modifications in the end-wall regions

For example, the maximum underturning at the tip is probably well correlated with the maximum loss coefficient at the tip. This is also true for the spanwise extent of the 3-D deviation and 3-D losses effects.

This approach for the 3-D loss correlation has been developed and the results are illustrated by Figure 10. It gives the maximum of the loss coefficient distribution at the shroud in function of the maximum deviation observed at the same location. The obtained relation is of the following form:

$$
\left(\varpi_{3 D}\right)_{\max , r t}=A \tanh \left(\bar{\Delta}_{5} \cdot \theta^{a} \cdot R^{b} \cdot \frac{\overline{\delta_{1}}}{A R}\right)^{c}
$$

Here again $A, a, b, c$ are the parameters of the distribution.

These parameters have been determined in the previous section to fit the parameters in the database. The prediction is considered to be sufficiently accurate.

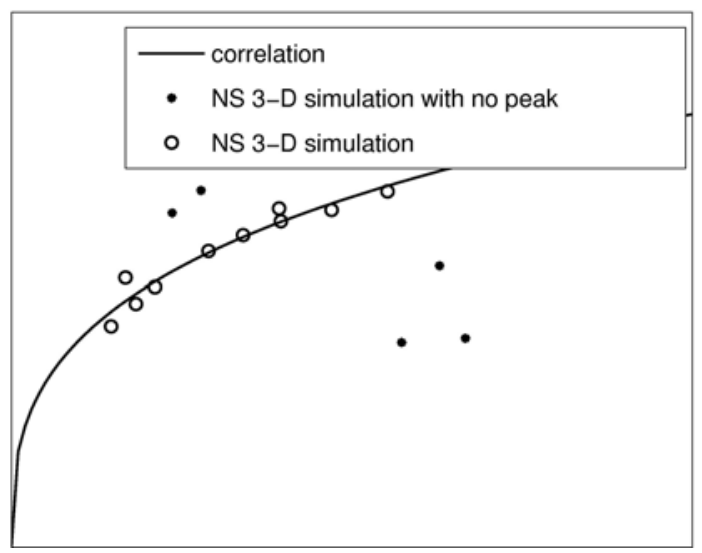

Figure 10: Maximum loss coefficient in function of the mamum deviation

\section{Conclusions}

The first approach solving the Euler equations does 
not need any refinement of the mesh in the vicinity of the hub and the shroud, resulting in moderate calculation efforts. However this first approach does not capture the complexity of the flow physics in the end-wall regions as the no-slip condition cannot be imposed, and therefore relies on empirical inputs that must be tuned or may suffer from a lack of generality.

The second approach solving the Navier-Stokes equations is less dependent on empirical inputs as more physics is included in the model, but at the price of a denser mesh and higher computational efforts. Figure 11 shows the improvement brought by theNavier-Stokes formulation over the Euler one for the radial distribution of the axial velocity at the outlet of the first rotor. More realistic distributions are indeed found with the Navier-Stokes model.

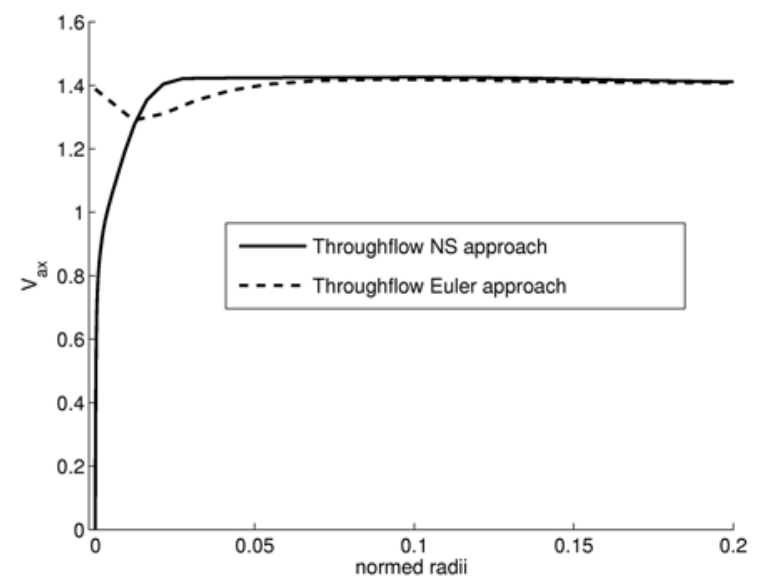

Figure 11: Comparison of non-dimensionalized axial velocity distributions

It is believed that the combination of both approaches, i.e. the Navier-Stokes approach with captured deviation and 3-D losses coefficient computed through calibrated functions (equation (18) for example) is a promising way to remove a part of the empiricism included in the throughflow model.

\section{References}

[1] S. Baralon, L.-E. Erikson and U. Hall. Viscous Throughflow Modelling of Transonic Compressors Using a
Time-Marching Finite-Volume Solver. Proceedings of the 13th International Symposium on Airbreathing Engines (ISABE), Chattanooga, 1997.

[2] J.D. Denton and W.N. Dawes. Computational Fluid Dynamics for Turbomachinery Design. In Developments in Turbomachinery Design, John Denton editor, Professional Engineering Publishing, 1999.

[3] R.P. Dring. Radial Transport and Momentum Exchange in an Axial Compressor. Transactions of the ASME Journal of Turbomachinery, 115: 477-486, 1993.

[4] S.J. Gallimore. Viscous Throughflow Modelling of Axial Compressor Bladerows using a Tangential Blade Force Hypothesis. ASME Paper 97-GT-415, 1997.

[5] W.M. Kšnig, D.K. Hennecke, and L. Fottner. Improved Blade Profile Loss and Deviation Angle Models for Advanced Transonic Compressor Blading : Part I - A Model for Subsonic Flow, Part II - A Model for Supersonic Flow. Transactions of the ASME Journal of Turbomachinery, 118: 73-80, 1996.

[6] E.W. Pfitzinger and W. Reiss. A New Concept for Loss and Deviation Prediction in Throughflow Calculations of Axial Flow Compressors. Proceedings of the 2nd European Turbomachinery Conference, Antwerpen, 1997.

[7] W.B. Roberts, G.K. Serovy and D.M. Sandercock. Modeling the 3-D Flow Effects on Deviation Angle for Axial Compressor Middle Stages. Journal of Engineering for Gas Turbines and Power, 108, 1986.

[8] W.B. Roberts, G.K. Serovy and D.M. Sandercock. Design Point Variation of 3-D Loss and Deviation for Axial Compressor Middle Stages. ASME Paper 88-GT-57, 1988.

[9] J. Simon and O. Léonard. A Throughflow Analysis Tool Based on the Navier-Stokes Equations. Proceedings of the 6th European Turbomachinery Conference, Lille, 2005.

[10] J.A. Storer and N.A. Cumpsty. Tip Leakage Flow in Axial Compressors. Transactions of the ASME Journal of Turbomachinery, 113: 252, 1991.

[11] A. Sturmayr and C. Hirsch. Shock Representation by Euler Throughflow Models and Comparison with Pitch-Averaged Navier-Stokes Solutions. ISABE 99-7281, 1999. 